\title{
IgG4-related aortitis/periaortitis and periarteritis: a distinct spectrum of IgG4- related disease
}

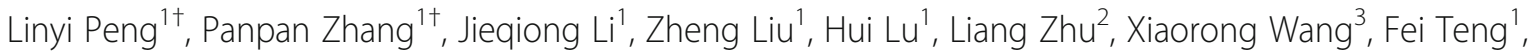
Xuemei $\mathrm{Li}^{4}$, Huifang Guo ${ }^{5}$, Yunyun Fei ${ }^{1}$, Wen Zhang ${ }^{1 *} \mathbb{D}$, Yan Zhao ${ }^{1}$, Xiaofeng Zeng ${ }^{1}$ and Fengchun Zhang ${ }^{1}$

\begin{abstract}
Background: Large vessels could be involved in immunoglobulin (Ig)-G4-related disease (IgG4-RD). This study aimed to clarify the clinical features and evaluate the treatment efficacy for lgG4-RD with aortitis/periaortitis and periarteritis (PAO/PA).

Methods: This study prospectively enrolled 587 patients with IgG4-RD with a follow-up time of more than 6 months. The distribution of IgG4-related PAO/PA was classified into four types: type 1, thoracic aorta; type 2a, abdominal aorta; type 2b, abdominal aorta and iliac artery; type 2c, iliac artery; type 3, thoracic and abdominal aorta; and type 4, other arteries. Patient's demographic data, clinical characteristics, laboratory parameters, and treatment efficacy were analyzed.

Results: Of 587 IgG4-RD patients, 89 (15.2\%) had PAO/PA. The average age was $58.3 \pm 11.1$ years, with male predominance (85.4\%). Vessels affected were as follows: abdominal aorta (83.1\%), iliac artery (70.8\%), thoracic aorta (13.5\%), and other vessels (13.5\%). The most prevalent distribution type of IgG4-related PAO/PA was type 2b, with 74 (83.1\%) patients, followed by type 2a, type 2c, type 3, and type 1. Fifty-five (61.8\%) PAO/PA patients had hydronephrosis, with renal insufficiency occurring in 43 (48.3\%), and 31 (34.8\%) PAO/PA patients had D-J stent drainage due to severe ureteral obstruction. After treatment with a glucocorticoid and immunosuppressants, $82 \%$ patients achieved remission with shrinking of the perivascular mass by more than $30 \%$.

Conclusions: IgG4-RD with PAO/PA was distinct from non-PAO/PA in demographic features, organ involvement distribution, inflammatory markers, and serum lgG4 and IgE. The most common affected vessel was the abdominal aorta, and most patients responded well with treatment.
\end{abstract}

Keywords: IgG4-related disease, Aortitis, Periaortitis, Periarteritis, Retroperitoneal fibrosis

\footnotetext{
* Correspondence: zhangwen91@sina.com

${ }^{\dagger}$ Linyi Peng and Panpan Zhang contributed equally to this work.

'Department of Rheumatology, Peking Union Medical College Hospital,

Chinese Academy of Medical Science \& Peking Union Medical College, Key

Laboratory of Rheumatology and Clinical Immunology, Ministry of Education

\& National Clinical Research Center for Dermatologic and Immunologic

Diseases (NCRC-DID), Beijing, China

Full list of author information is available at the end of the article
}

(c) The Author(s). 2020 Open Access This article is licensed under a Creative Commons Attribution 4.0 International License, which permits use, sharing, adaptation, distribution and reproduction in any medium or format, as long as you give appropriate credit to the original author(s) and the source, provide a link to the Creative Commons licence, and indicate if changes were made. The images or other third party material in this article are included in the article's Creative Commons licence, unless indicated otherwise in a credit line to the material. If material is not included in the article's Creative Commons licence and your intended use is not permitted by statutory regulation or exceeds the permitted use, you will need to obtain permission directly from the copyright holder. To view a copy of this licence, visit http://creativecommons.org/licenses/by/4.0/ The Creative Commons Public Domain Dedication waiver (http://creativecommons.org/publicdomain/zero/1.0/) applies to the data made available in this article, unless otherwise stated in a credit line to the data. 


\section{Background}

Immunoglobulin (Ig)-G4-related disease (IgG4-RD) is a fibro-inflammatory condition characterized by tumorlike swelling of affected organs, with elevated serum IgG4 and massive infiltration of lymphocytes and plasma cells in involved organs [1-5]. It is a highly heterogeneous disease entity that could affect nearly any organ and often present with multiorgan involvement [6], and the most affected tissues are the lacrimal gland, submandibular gland, lymph node, and pancreas [6-8]. Evidence suggested that IgG4-RD could affect various organs including the vascular system as aortitis/periaortitis/periarteritis (PAO/PA) [9-12]; additionally, the most frequently involved blood vessel is the aorta, and a medium-sized artery, such as the iliac artery and carotid artery, may be a potential target [5].

$\mathrm{PAO} / \mathrm{PA}$ was reported to be present in $10-30 \%$ of overall IgG4-RD, and they may appear as an isolated lesion of IgG4-RD. Studies had investigated the histopathological diagnosis of the vascular involvement of IgG4-RD, vessel distributions, concomitant non-vascular lesions, treatment efficacy, or potential etiology $[5,10$, 13, 14]. However, the lack of large prospective studies may hinder our understanding of the complete clinical picture of IgG4-related PAO/PA because of its heterogeneity. Moreover, early diagnosis and treatment are essential to minimizing irreversible organ damage or unnecessary surgical intervention [6], such as aneurysmal dilation or rupture of the aorta, or irreversible renal dysfunction due to the obstruction of ureters and hydronephrosis. Therefore, to further understand and achieve better management of IgG4-related PAO/ PA, we aimed to analyze the clinical patterns of IgG4related $\mathrm{PAO} / \mathrm{PA}$, distribution of affected vessels, and treatment efficacy.

\section{Methods}

\section{Inclusion and exclusion criteria}

In our prospective cohort of IgG4-RD carried out in the Peking Union Medical College Hospital (registered as ClinicalTrials.gov ID: NCT01670695), 587 patients were enrolled from January 2011 to September 2018, fulfilling the 2011 comprehensive diagnostic criteria $[15,16]$. Each IgG4-RD patient had follow-up time of more than 6 months. The diagnosis of IgG4-RD was based on the following criteria: (1) a clinical examination showing characteristic diffuse/localized swelling or masses in single or multiple organs, (2) an elevated serum IgG4 concentration (>135 mg/dL), and (3) a histopathologic examination showing (a) marked lymphocytic and plasma cell infiltration and fibrosis or (b) infiltration of IgG4+ plasma cells (a ratio of IgG4+/IgG+ cells $>40 \%$ and $>10$ IgG4+ plasma cells per high power field). Patients with other autoimmune diseases, active/severe infection, and malignant disease were excluded. Patients combined with antineutrophil cytoplasmic antibody-associated vasculitis were excluded. Patients' affected organs and evaluation of treatment efficacy were determined by clinical symptoms, physical examinations, histological pathology, and imaging, including ultrasonography, computed tomography (CT), magnetic resonance imaging (MRI), or positron emission tomography/computed tomography (PET/CT). Allergy history information was collected using the criteria from the European Academy of Allergy and Clinical Immunology. The study was conducted in compliance with the Declaration of Helsinki and was approved by the Ethics Committee of Peking Union Medical College Hospital (number S-442). All patients signed written informed consent.

\section{Detection of PAO/PA by CT, MRI or PET-CT}

A patient was diagnosed with $\mathrm{PAO} / \mathrm{PA}$ if one or more of the following conditions are present: (1) vessel wall thickening, (2) vessel wall enhancement on contrast imaging, (3) soft tissues around blood vessels with circumferential enhancement on CT or MRI, (4) and ${ }^{18} \mathrm{flu}$ deoxyglucose avidity within a vessel wall or perivascular region on $\mathrm{PET} / \mathrm{CT}[5,9]$. Improvement of $\mathrm{PAO} / \mathrm{PA}$ was defined as shrinking of perivascular soft tissues and reduced vessel wall thickness by averaging the 2 dimensions of greatest change on CT. Luminal dilatation at the time of periaortic/periarterial lesion diagnosis was defined as aneurysm, in which the luminal diameter was $>1.5$ times wider than normal $(>45 \mathrm{~mm}$ at the thoracic aorta and $>30 \mathrm{~mm}$ at the infra-renal abdominal aorta) $[17,18]$. This study included $89 \mathrm{PAO} / \mathrm{PA}$ patients; 8 patients with retroperitoneum lesion but without vessels affected were excluded, and patients with malignant tumor during follow-up were excluded. The treatment efficacy was evaluated by clinical characteristics, laboratory parameters, and CT scans.

For 43 patients with IgG4-related PAO/PA with renal impairment, the estimated glomerular filtration rate (eGFR) was calculated before and after 3 months of treatment using the Chronic Kidney DiseaseEpidemiology Collaboration formula. Removal time of the ureteral stent was decided by consensus among rheumatologists and urologists after determining whether the soft tissue around the ureter was reduced, ureteral compression was relieved, and hydronephrosis and renal function improved [19]. We monitored renal function and ultrasonography of the kidney every 2 weeks during the first 2 months after stent removal. If renal function was stable and hydronephrosis was not aggravated, stent removal was considered successful.

Based on Ozawa et al.'s study of vessel distribution [5] and our clinical experience, PAO/PA in our study was 
classified into four types according to the distribution of affected vessels: type 1 , thoracic aorta; type 2 , abdominal aorta and iliac artery involved, including type $2 \mathrm{a}$, abdominal aorta, type $2 \mathrm{~b}$, abdominal aorta and iliac artery, type 2c, iliac artery; type 3 , thoracic and abdominal aorta; and type 4, other arteries (Fig. 1).

\section{Clinical data and laboratory parameters}

Patients' data including age, sex, disease duration, history of allergy, treatment strategy, symptom onset, organs affected, and follow-up time were collected. Disease duration refers to the interval between symptom onset and diagnosis. The IgG4-RD responder index (RI) (2015 version) at baseline and each follow-up was evaluated [20]. Laboratory parameters included routine blood analysis; liver function; kidney function; serum IgG, A, and M; serum IgG subclass; total serum IgE; rheumatoid factor, C3 and C4, erythrocyte sedimentation rate (ESR), and hypersensitive $\mathrm{C}$-reactive protein (hsCRP) tests.

\section{Flow cytometry of $\mathrm{CD} 19^{+} \mathrm{CD} 24^{-} \mathrm{CD} 38^{\text {hi }}$ plasmablast/ plasma cells}

Peripheral blood mononuclear cells from IgG4-RD patients were separated by Ficoll gradient centrifugation. B cell subpopulations were stained with $\mathrm{PE}-\mathrm{Cy} 7$-conjugated anti-CD19, FITC-conjugated anti-CD24, and APC- conjugated anti-CD38 (BD Bioscience, USA). Plasmablast/ plasma cell was defined as $\mathrm{CD} 19^{+} \mathrm{CD} 24^{-} \mathrm{CD} 38^{\text {hi }}$. Altogether, 93 patients tested $\mathrm{CD} 19^{+} \mathrm{CD} 24^{-} \mathrm{CD} 38^{\text {hi }}$ plasmablast/plasma cells at baseline, including 18 IgG4-RD $\mathrm{PAO} / \mathrm{PA}$ patients and 75 non-PAO/PA patients.

\section{Treatment efficacy assessment}

According to the comparison CT scans before treatment and at 3 months after treatment, the reduction of soft tissue around affected vessels was categorized into three types: $0-30 \%, 31-70 \%$, and $>70 \%$.

Treatment response was assessed by evaluating the changes in IgG4-RD RI scores and was divided into complete response (CR), partial response (PR), and no effect (NE, including no improvement or exacerbation). IgG4-RD RI scores $<3$ and decline of $\geq 2$ were recognized as CR; IgG4-RD RI score decline of $\geq 2$ but remained $\geq 3$ were recognized as PR. If patients' IgG4RD RI score was 3 points at the beginning, PR was considered a 1-point decrease after the therapy. Patients lacking apparent changes in mass sizes and/or clinical manifestations and IgG4-RD RI score decline of $<2$ were considered NE [21]. Clinical relapse was defined as reappearance of clinical symptoms or imaging findings were worsened with or without elevated serum IgG4 levels [21, 22].

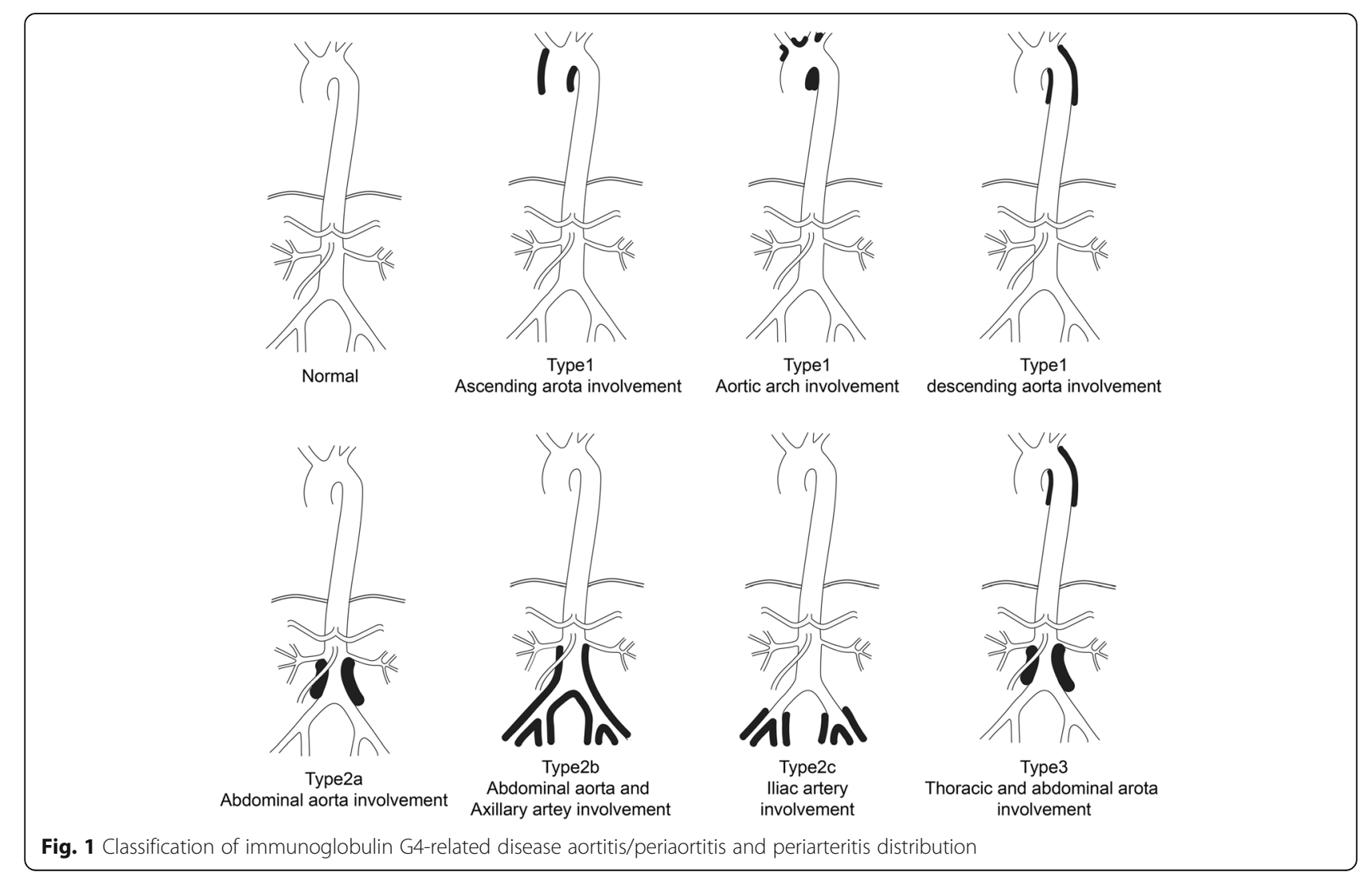




\section{Statistical methods}

Statistical analyses were performed using the IBM SPSS Statistics version 24.0 software (IBM, Armonk, NY, USA), Adobe Illustrator CC 2014 (Adobe Systems, San Jose, CA, USA), and the Prism software version 6.1 (GraphPad Software, La Jolla, CA, USA). Data were reported as means \pm standard deviation or median (quartile 1 [Q1]-quartile 3 [Q3]). Normally distributed data between two groups were analyzed using independentsamples $t$ tests or paired-samples $t$ tests, and a one-way analysis of variance was used to compare groups. Categorical data were analyzed using the chi-square test or Fisher's exact tests, while non-normally distributed data were analyzed using the rank sum test. A two-tailed $P$ value $<0.05$ was considered statistically significant.

\section{Results}

Demographic characteristics of IgG4-RD with PAO/PA

Among 587 IgG4-RD patients, 89 patients (76 men and 13 women) were included in the IgG4-related PAO/PA group and 498 patients were included in the non-PAO/ PA group. Of the IgG4-RD patients with $\mathrm{PAO} / \mathrm{PA}, 24$ (27.0\%) had PAO/PA alone, while the other patients $(n=65,73.0 \%)$ had multiple organ involvement. Demographic features of IgG4-RD with/without PAO/PA are shown in Table 1. The age of PAO/PA patients was $58.3 \pm 11.1$ years, with a male/female ratio of $5.85 / 1$. The IgG4-RD RI was $10.8 \pm 5.3$ at baseline. Male patients $(59.5 \pm 9.7$ years $)$ were older than female patients $(51.3 \pm$ 16.1 years $)$ at disease onset $(P=0.015)$. Moreover, 25 (28.1\%) PAO/PA patients had an allergic history. The median follow-up time was $30(15,52)$ months.

We compared the prevalence of allergic diseases in IgG4-PAO/PO patients with/without extra-glandular involvement. The results showed that allergic diseases were more common in patients with lacrimal and/or salivary gland lesions than in those without $(53.6 \%$ versus $16.4 \%, P<0.001)$.

Table 1 Demographic features of IgG4-RD with/without PAO/ PA

\begin{tabular}{|c|c|c|c|}
\hline Demographic features & $\begin{array}{l}\mathrm{PAO} / \mathrm{PA} \\
(\boldsymbol{n}=89)\end{array}$ & $\begin{array}{l}\text { Non-PAO/PA } \\
(\boldsymbol{n}=498)\end{array}$ & $\boldsymbol{P}$ value \\
\hline Age (years) & $58.3 \pm 11.1$ & $52.6 \pm 13.8$ & $<0.001^{*}$ \\
\hline Male/female ratio & $5.85 / 1$ & $1.35 / 1$ & $<0.001^{*}$ \\
\hline $\begin{array}{l}\text { Disease duration } \\
\text { (month), M (Q1-Q3) }\end{array}$ & $6(2.5-36)$ & $12(6-36)$ & $<0.001^{*}$ \\
\hline History of allergy $(n, \%)$ & $25(28.1)$ & $267(53.6)$ & $<0.001^{*}$ \\
\hline lgG4-RD RI & $10.8 \pm 5.3$ & $9.8 \pm 5.2$ & 0.103 \\
\hline Number of organs involved & $2.9 \pm 1.9$ & $3.0 \pm 1.7$ & 0.126 \\
\hline $\begin{array}{l}\text { Patients with single organ } \\
\text { involved }(n, \%)\end{array}$ & $24(27.0)$ & $66(13.3)$ & $0.001^{*}$ \\
\hline
\end{tabular}

*Statistical significance
Of 89 patients with $\mathrm{PAO} / \mathrm{PA}, 35$ (39.3\%) patients were diagnosed as having definite IgG4-RD, 1 (1.1\%) was probable, and $53(59.6 \%)$ patients were possible. Nine (10.1\%) patients underwent perivascular mass biopsy, while the rest of the patients underwent biopsy of other involved organs, including the submandibular gland, lacrimal gland, kidney, lymph node, and lung.

\section{Symptoms at disease onset in IgG4-RD patients with PAO/PA}

Symptoms at disease onset in IgG4-RD patients with $\mathrm{PAO} / \mathrm{PA}$ are shown in Table 2. Pain was the most prevalent symptom observed; among all patients, 57 (64.0\%) patients had onset symptoms of back pain (32, $36.0 \%)$ and abdominal pain (25, 28.1\%). Fourteen patients (15.7\%) had lower limb edema. Other onset symptoms included lymph node swelling (22, 24.7\%),

Table 2 Onset symptoms and organs involvement of IgG4-RD patients with/without PAO/PA

\begin{tabular}{llll}
\hline $\begin{array}{l}\text { Symptoms and organs affected } \\
\text { at baseline }\end{array}$ & PAO/PA & Non-PAO/PA & $P$ value \\
\hline Symptoms at disease onset $(n, \%)$ & & & \\
Back pain & $32(36)$ & $21(4.2)$ & $<0.001^{*}$ \\
Lymph node swelling & $22(24.7)$ & $137(27.5)$ & 0.575 \\
Abdominal pain & $25(28.1)$ & $79(15.9)$ & $0.005^{*}$ \\
Submandibular gland enlargement & $18(20.2)$ & $213(42.8)$ & $<0.001^{*}$ \\
Lacrimal gland enlargement & $10(11.2)$ & $227(45.6)$ & $<0.001^{*}$ \\
Lower limb edema & $14(15.7)$ & $8(1.6)$ & $<0.001^{*}$ \\
Cough & $11(12.4)$ & $53(10.6)$ & 0.632 \\
Nausea and vomiting & $12(11.1)$ & $42(8.4)$ & 0.129 \\
Jaundice & $8(9.0)$ & $72(14.5)$ & 0.166 \\
Parotid gland enlargement & $5(5.6)$ & $66(13.3)$ & $0.042^{*}$ \\
Nasal congestion & $4(4.5)$ & $106(21.3)$ & $<0.001^{*}$ \\
Itching & $5(5.6)$ & $45(9.0)$ & 0.287 \\
Organs affected (n, \%) & & & \\
Lymph node & $33(37.1)$ & $219(44)$ & 0.674 \\
Submandibular gland & $24(27.0)$ & $259(52)$ & $<0.001^{*}$ \\
Pancreas & $26(29.2)$ & $175(35.5)$ & 0.278 \\
Lung & $15(16.9)$ & $117(23.5)$ & 0.167 \\
Lacrimal gland & $11(12.4)$ & $266(53.4)$ & $<0.001^{*}$ \\
Parotid gland & $9(10.1)$ & $90(18.1)$ & 0.065 \\
Bile duct & $11(12.4)$ & $99(19.9)$ & 0.094 \\
Paranasal sinus & $8(9.0)$ & $155(31.1)$ & $<0.001^{*}$ \\
Prostate & $12(15.8)$ & $39(13.6)$ & 0.632 \\
Kidney & $6(6.7)$ & $44(8.8)$ & 0.515 \\
Thyroid & $2(2.2)$ & $21(4.2)$ & 0.378 \\
Pituitary & $28.6)$ & 0.778 \\
\hline & 0.071 \\
\hline
\end{tabular}


submandibular gland enlargement (18, 20.2\%), cough (11, 12.4\%), lacrimal gland enlargement (10, 11.2\%), jaundice $(8,9.0 \%)$, parotid gland enlargement $(5,5.6 \%)$, and nasal congestion $(4,4.5 \%)$.

\section{Laboratory parameters of IgG4-RD patients with PAO/PA}

Among IgG4-RD patients with $\mathrm{PAO} / \mathrm{PA}$, the serum creatinine level increased in $43(48.3 \%)$ patients (131 $[117,179] \mu \mathrm{mol} / \mathrm{L})$ (Table 3), including 39 (51.3\%) male patients and $4(30.8 \%)$ female patients. ESR and hsCRP values were $44(18-75) \mathrm{mm} / \mathrm{h}$ and $6.72(2.14-24.65)$ $\mathrm{mg} / \mathrm{L}$, respectively. Serum IgG, IgG4, and T-IgE levels were $19.88 \pm 8.20 \mathrm{~g} / \mathrm{L}, 4240(2015,7730) \mathrm{mg} / \mathrm{L}$, and 170 $(95.3,463.5) \mathrm{KU} / \mathrm{L}$, respectively.

\section{Comparison of IgG4-RD patients with/without PAO/PA} Compared with non-PAO/PA patients, $\mathrm{PAO} / \mathrm{PA}$ patients were older at disease onset, had a higher male/female ratio, but had shorter disease duration and lower percentage of an allergic history $(P<0.001, P<0.001$, $P<0.001$, and $P<0.001$, respectively; Table 1$)$. The number of organs involved and IgG4-RD RI were comparable in patients with/without $\mathrm{PAO} / \mathrm{PA}$. However, patients with $\mathrm{PAO} / \mathrm{PA}$ had a higher percentage of single organ involvement than those without PAO/PA $(P<0.001)$.
Patients with $\mathrm{PAO} / \mathrm{PA}$ had a higher percentage of back pain, abdominal pain, and lower limb edema than those with $\mathrm{PAO} / \mathrm{PA}(4.2 \%[P<0.001], 15.9 \%[P=0.005]$, and $1.6 \%[P<0.001]$, respectively). In contrast, patients with $\mathrm{PAO} / \mathrm{PA}$ had a lower percentage of submandibular gland enlargement, lacrimal gland enlargement, parotid gland enlargement, and nasal congestion than those without $\mathrm{PAO} / \mathrm{PA} \quad(42.8 \%[P<0.001], 45.6 \% \quad[P<0.001], 13.3 \%$ $[P=0.042]$, and $21.3 \%[P<0.001]$, respectively) (Table 2 ). Consistent with onset symptoms, patients with $\mathrm{PAO} / \mathrm{PA}$ had a lower percentage of submandibular gland, lacrimal gland, and paranasal involvement (all $P<0.001$ ) (Table 2).

Compared to patients with $\mathrm{PAO} / \mathrm{PA}$, those with PAO/PA had higher levels of ESR, hsCRP, and IgA $(P<0.0001, \quad P<0.0001$, and $P=0.02$, respectively $)$ (Table 3). However, patients with IgG4-related PAO/ PA had lower levels of serum IgG4 and IgE than those without IgG4-related PAO/PA (8310 [3250, 17, 075] $\mathrm{mg} / \mathrm{L}, 332$ [119, 720.5] KU/L; $P<0.0001$ and $P=$ 0.025 , respectively) (Table 3). Additionally, serum IgG4 levels were higher in patients with $\mathrm{PAO} / \mathrm{PA}$ and other organs affected $(5270[2395,11,910] \mathrm{mg} / \mathrm{L})$ than in those with only PAO/PA $(2418[1583,4638] \mathrm{mg} / \mathrm{L})$ $(P=0.001)$.

Table 3 Laboratory parameters of IgG4-RD patients with/without PAO/PA

\begin{tabular}{|c|c|c|c|}
\hline Parameters & $\mathrm{PAO} / \mathrm{PA}(\boldsymbol{n}=89)$ & Non-PAO/PA $(\boldsymbol{n}=498)$ & $\boldsymbol{P}$ value \\
\hline $\mathrm{HgB}(\mathrm{g} / \mathrm{L})$ & $127 \pm 21$ & $135 \pm 19$ & $<0.001^{*}$ \\
\hline WBC $\left(10^{9} / \mathrm{L}\right)$ & $7.9 \pm 2.8$ & $7.15 \pm 2.55$ & $0.014^{*}$ \\
\hline $\operatorname{PLT}\left(10^{9} / \mathrm{L}\right)$ & $240 \pm 87$ & $238 \pm 89$ & 0.862 \\
\hline Eos\% elevation (\%) & 22.5 & 32.4 & 0.077 \\
\hline ESR (mm/h), M (Q1-Q3) & $44(18-75)$ & $16(7-40)$ & $<0.0001^{*}$ \\
\hline Elevation of ESR $(n, \%)$ & $61(77.1 \%, 61 / 70)$ & $205(42.0 \%, 205 / 488)$ & $<0.001^{*}$ \\
\hline hsCRP (mg/L), M (Q1-Q3) & $6.72(2.14-24.65)$ & $1.78(0.72-5.12)$ & $<0.0001^{*}$ \\
\hline Elevation of hsCRP $(n, \%)$ & $56(70 \%, 56 / 80)$ & $150(37.5 \%, 150 / 400)$ & $<0.001^{*}$ \\
\hline $\lg G(g / L)$ & $19.88 \pm 8.20$ & $21.28 \pm 14.46$ & 0.292 \\
\hline $\lg A(g / L)$ & $2.53 \pm 1.13$ & $2.16 \pm 1.29$ & $0.02^{*}$ \\
\hline $\lg M(g / L), M(Q 1-Q 3)$ & $0.88(0.56-1.14)$ & $0.77(0.54-1.19)$ & 0.573 \\
\hline lgG1 (mg/L), M (Q1-Q3) & $9355(7928-11,325)$ & $8665(7013-10,600)$ & 0.03 \\
\hline lgG2 (mg/L), M (Q1-Q3) & $5705(4255-7350)$ & $5595(4290-7520)$ & 0.873 \\
\hline lgG3 (mg/L), M (Q1-Q3) & $461(221-923)$ & $439(253-841)$ & 0.802 \\
\hline lgG4 (mg/L), M (Q1-Q3) & $4240(2015-7730)$ & $8310(3250-17,075)$ & $<0.0001^{*}$ \\
\hline T-IgE (KU/L), M (Q1-Q3) & $170(95.3-463.5)$ & $332(119-720.5)$ & $0.025^{*}$ \\
\hline $\mathrm{Cr}(\mu \mathrm{mol} / \mathrm{L})$ & $98.5(73-131.3)$ & $67(57.7-78)$ & $<0.0001^{*}$ \\
\hline Elevation of $\mathrm{Cr}(n, \%)$ & $38(50)$ & $14(2.8)$ & $<0.001^{*}$ \\
\hline C3 (g/L) & $1.018 \pm 0.322$ & $0.948 \pm 0.330$ & 0.725 \\
\hline $\mathrm{C} 4(\mathrm{~g} / \mathrm{L})$ & $0.217 \pm 0.112$ & $0.173 \pm 0.102$ & $0.004^{*}$ \\
\hline
\end{tabular}

M (Q1-Q3) represented median (quartile 1to quartile 3) 


\section{$\mathrm{CD} 19^{+} \mathrm{CD} 24^{-} \mathrm{CD} 38^{\text {hi }}$ plasmablast/plasma cells}

Overall, 93 patients showed $\mathrm{CD} 19^{+} \mathrm{CD} 24^{-} \mathrm{CD} 38^{\text {hi }}$ plasmablast/plasma cells at baseline. No statistical significant difference in $\mathrm{CD} 19^{+} \mathrm{CD} 24^{-} \mathrm{CD} 38^{\text {hi }}$ plasmablast/ plasma cell was found between $\mathrm{PAO} / \mathrm{PA}$ and non$\mathrm{PAO} / \mathrm{PA}$ patients. However, the percentage of $\mathrm{CD} 19^{+} \mathrm{CD} 24^{-} \mathrm{CD} 38^{\text {hi }}$ plasmablast/plasma cells was lower in patients with only $\mathrm{PAO} / \mathrm{PA}$ (2.22\% [Q1-Q3, $1.87-5.90 \%]$ ) than in those with $\mathrm{PAO} / \mathrm{PA}$ and other organ involvement (8.5\% [Q1-Q3, 4.52-16.90\%]) or non-PAO/PA $\quad(5.58 \% \quad[\mathrm{Q} 1-\mathrm{Q} 3,2.99-10.50 \%]) \quad(P=$ 0.015 and $P=0.023$, respectively).

\section{Vessels distribution of IgG4-related PAO/PA}

Characteristic imaging findings IgG4-related PAO/PA are shown in Fig. 2. Of 89 IgG4-RD patients with PAO/ PA, the abdominal aorta was the most affected vessel $(74,83.1 \%)$, followed by the iliac artery $(63,70.8 \%)$, thoracic aorta (12, 13.5\%), and other vessels, including the superior mesenteric artery $(6,6.7 \%)$, renal artery $(6$, $6.7 \%)$, common carotid artery (3,3.4\%), and subclavian artery (2, 2.2\%), revealing vascular stenosis in 3 patients. Beside soft tissue around the vessels, 27 patients had calcification of the aortic wall, $22.5 \%$ had diffuse thickening of the abdominal aortic wall, and $10.1 \%$ had aneurysmal dilation of the aorta (Table 4). Abdominal aorta and iliac artery lesions were more common in male patients than in female patients $(P=0.024$ and $P=0.035$, respectively;
Table 4). However, the ratio of patients with thoracic aorta and other large vessels affected was higher in female patients than in male patients $(P=0.048$ and $P=$ 0.004 , respectively; Table 4). Moreover, female patients had a lower percentage of aortic wall calcification than male patients $(P=0.047$; Table 4$)$.

According to the distribution of IgG4-related PAO/ PA, type 2, involvement of abdominal aorta and iliac artery, was the most prevalent $(74,83.1 \%)$, especially type $2 \mathrm{~b}(52,58.4 \%)$, followed by type $2 \mathrm{a}(15,16.9 \%)$, type $2 \mathrm{c}$ (7, 7.9\%), type $3(7,7.9 \%)$, type $1(5,5.6 \%)$, and type 4 ( 3 , $3.4 \%$ ) (Table 4). Additionally, no statistical significance in vessel distribution was found between patients with $\mathrm{PAO} / \mathrm{PA}$ alone and those with PAO/PA and other organ involvement.

Further, 55 (61.8\%) patients with PAO/PA had hydronephrosis, including $47(61.8 \%)$ male patients and 8 (61.5\%) female patients. Forty-three (48.3\%) patients developed impairment of renal function caused by ureteral obstruction. Of patients with severe obstruction, double $\mathrm{J}(\mathrm{D}-\mathrm{J})$ stent drainage was the first option to relieve obstruction, and 31 (34.8\%) PAO/PA patients had D-J stent drainage. With regard to other organs affected, the rates of lymph node, pancreas, submandibular gland, lung, prostate, bile duct, lacrimal gland, parotid gland, paranasal sinus, and thyroid gland involvement were $37.1 \%$, $29.2 \%, 27.0 \%, 16.9 \%, 15.8 \%, 12.4 \%, 12.4 \%, 10.1 \%, 9.0 \%$, and $2.2 \%$, respectively (Table 2).

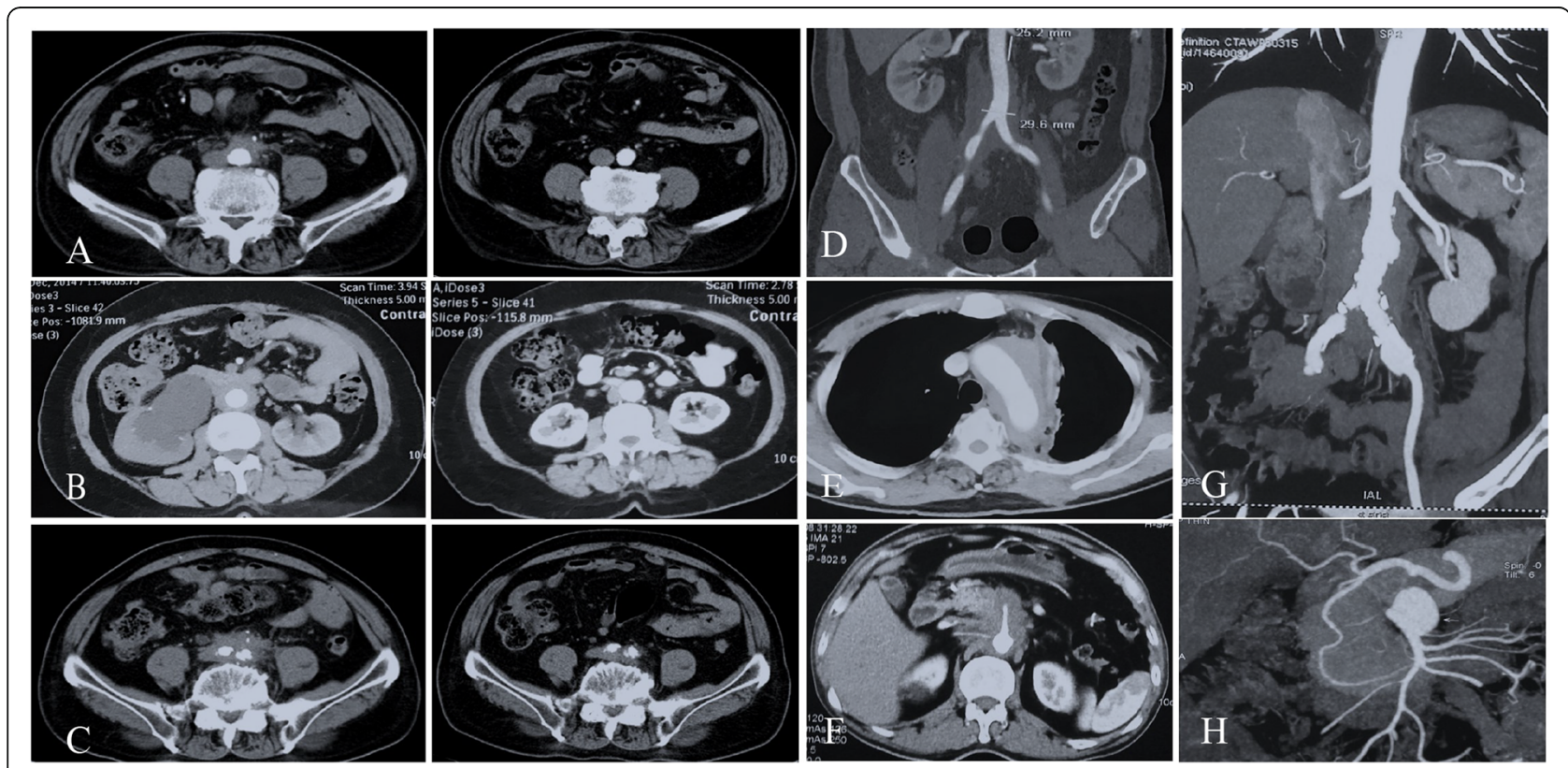

Fig. 2 Characteristic imaging findings of immunoglobulin G4-related disease aortitis/periaortitis and periarteritis. a A 69-year-old man with periaortitis before and after treatment. b A 52-year-old woman with periaortitis and hydronephrosis before and after treatment. c The affected iliac artery before and after treatment. $\mathbf{d}$, e A 54-year-old man with thoracic aorta, abdominal, and iliac artery involvement. f Superior mesenteric artery involvement in a 64-year-old man. $\mathbf{g}, \mathbf{h}$ Aneurysmal dilation of the abdominal aorta and superior mesenteric artery 
Table 4 Vascular distribution of IgG4-RD with PAO/PA

\begin{tabular}{|c|c|c|c|c|}
\hline Vessels affected $(\boldsymbol{n}, \%)$ & Total $(\boldsymbol{n}=89)$ & Male $(\boldsymbol{n}=76)$ & Female $(\boldsymbol{n}=13)$ & $\boldsymbol{P}$ value \\
\hline Abdominal aorta & $74(83.1)$ & $66(86.8)$ & $8(61.5)$ & $0.024^{*}$ \\
\hline \|liac artery & $63(70.8)$ & $57(75.0)$ & $6(46.2)$ & $0.035^{*}$ \\
\hline Thoracic aorta & $12(13.5)$ & $8(10.5)$ & $4(30.8)$ & $0.048^{*}$ \\
\hline Other vessels & $12(13.5)$ & $7(9.2)$ & $5(38.5)$ & $0.004^{*}$ \\
\hline Calcification of vessel wall & $27(30.3)$ & $26(34.2)$ & $1(7.7)$ & $0.047^{*}$ \\
\hline Diffuse thickening of the abdominal aortic wall & $20(22.5)$ & $16(21.1)$ & $4(30.8)$ & 0.438 \\
\hline Aneurysm & $9(10.1)$ & $9(11.8)$ & $0(0.0)$ & 0.346 \\
\hline Type 1 & $5(5.6)$ & $2(2.6)$ & $3(23.1)$ & $0.003^{*}$ \\
\hline Type 2 & $74(83.1)$ & $66(86.8)$ & $8(61.5)$ & $0.024^{*}$ \\
\hline Type 2a & 15 (16.9) & $13(17.1)$ & $2(15.4)$ & 1.000 \\
\hline Type 2b & $52(58.4)$ & 47 (83.9) & $5(38.5)$ & 0.114 \\
\hline Type 2c & $7(7.9)$ & $6(7.9)$ & $1(7.7)$ & 1.000 \\
\hline Type 3 & $7(7.9)$ & $6(7.9)$ & $1(7.7)$ & 0.980 \\
\hline Type 4 & $3(3.4)$ & $2(2.6)$ & $1(7.7)$ & 0.381 \\
\hline
\end{tabular}

*Statistical significance

\section{Clinical features among the four types of vessel involvement groups}

We compared the clinical characteristics at baseline of IgG4-related PAO/PA patients with different vessel distributions (type 1 , type $2 \mathrm{a}$, type $2 \mathrm{~b}$, type $2 \mathrm{c}$, type 3 , and type 4) in supplementary table 1 . The results indicated that male patients were predominant in type 2 than in type $1(P=0.018)$. Among all the groups, IgG4-RD RI was highest in type $2 \mathrm{a}(P=0.047)$. The percentage of creatinine elevation caused by ureter obstruction was much higher in type 2 and type 3 than in the others. There was no statistical significance in the other clinical features among the four groups.

\section{Treatment efficacy in patients with PAO/PA}

Patients with $\mathrm{PAO} / \mathrm{PA}$ were treated with glucocorticoids (GCs) or GCs combined with immunosuppressant agents (GCs plus immunosuppressant agent).

Except for 18 (20.2\%) patients who received GC monotherapy, the other patients were treated with GCs plus cyclophosphamide (CYC) $(n=52,58.4 \%)$, GCs plus mycophenolate mofetil (MMF) $(n=18,20.2 \%)$, and GCs plus leflunomide $(n=1,1.1 \%)$. Forty-one $(46.1 \%)$ patients received combined treatment with tamoxifen.

After 6 months of treatment, 34 (38.2\%) patients achieved reduction of perivascular soft tissues $>70 \%, 39$ (43.8\%) achieved reduction between 31 and 70\%, and 16 (18.0\%) had reduction $<30 \%$. Compared with male patients with $\mathrm{PAO} / \mathrm{PA}$, a higher percentage of female patients had reduction of perivascular soft tissues $<30 \%$ $(P=0.01)$. In $31(34.8 \%)$ patients who had D-J stent drainage, $22(71.0 \%)$ patients had successful stent extubation, and the median time of extubation was 6 (313.5) months. Of 43 patients with renal function impairment, $72.1 \%$ patients with renal insufficiency at baseline showed normalization of the serum creatinine during the follow-up. Twelve (27.9\%) patients' serum creatinine level decreased but remained above the normal range. The eGFR before treatment was $46.0 \pm 18.0$ $\mathrm{ml} / \mathrm{min} / 1.73 \mathrm{~m}^{2}$, and it increased to $66.7 \pm 18.8 \mathrm{ml} / \mathrm{min} /$ $1.73 \mathrm{~m}^{2}$ after therapy (supplementary figure 1 ).

Nine $(10.1 \%)$ patients who had aneurysmal dilatation at baseline received initial dose of GCs $(0.5 \mathrm{mg} / \mathrm{kg}) \mathrm{com}$ bined with immunosuppressant agent. We monitored changes of the dilated vessels by CT scan every 3 6 months in the first year after diagnosis. After treatment, there was no significant change in the dilatation scope and dilated diameter of PAO/PA. No patient experienced aneurysmal rupture.

The IgG4-RD RI and ESR, hsCRP, serum IgG4, and IgE levels reduced significantly after treatment. The serum IgG4 level returned to the normal range in 57.3\% of patients with $\mathrm{PAO} / \mathrm{PA}$, and the serum IgE level returned to the normal range in $33.7 \%$ of patients after 6 months of treatment.

Five (5.6\%) patients relapsed during the follow-up with a median recurrence time of 21 (15.5-33) months; all of them had two or multiple organs involved at baseline and relapsed in other organs beyond the blood vessels. No significant difference in the relapse rate was found between male and female patients.

Comparison between IgG4-related PAO/PA patients with shrinkage of soft tissues around vessels $>70 \%$ and $<30 \%$ In order to clarify the differences of IgG4-related PAO/ PA patients who had significantly different treatment efficacy, we compared the clinical features between the more than $70 \%$ responder group (group A) and less than 
$30 \%$ responder group (group B) (supplementary table 2). Patients in group A were older and more predominantly male than those in group B. Regarding organ involvement, the percentage of lymphadenopathy was higher in group A than in group $\mathrm{B}(P=0.01)$, while other organs involvement was comparable between group $\mathrm{A}$ and group $B$. The IgG3 level was higher in group A than in group B $(P=0.006)$. There was no statistical significance in other clinical features between the two groups.

\section{Discussion}

To our knowledge, this is the largest prospective cohort of IgG4-RD patients with $\mathrm{PAO} / \mathrm{PA}$ who were compared to patients without $\mathrm{PAO} / \mathrm{PA}$. We compared the clinical manifestations in IgG4-RD with or without PAO/PA in a large prospective cohort from China. In addition, the treatment efficacy in PAO/PA patients was evaluated.

IgG4-related $\mathrm{PAO} / \mathrm{PA}$ was associated with male predominance and could affect multiple organs [10-12], and it affects $60-85 \%$ of male patients [10, 13, 23]; thus, it is different from Takayasu arthritis (TA) with a young female predominance. Male PAO/PA patients were older at disease onset than female patients. Consistent with other reported IgG4-related PAO/PA, gender has a strong influence on the pattern of vascular involvement and consequently on clinical presentation; women have a higher involvement of supradiaphragmatic vessels, whereas men have abdominal vessel involvement $[9,13,24]$.

According to allergy, a substantial percentage of IgG4$\mathrm{RD}$ patients had histories of allergy [8], the percentage of allergy was higher in patients with IgG4-related dacryoadenitis/sialadenitis (DS) than in those with nonIgG4 DS [7], and non-PAO/PA patients had a higher percentage of allergy than $\mathrm{PAO} / \mathrm{PA}$ patients [5]. Our result also showed that allergic diseases were significantly more common in patients with lacrimal and/or salivary gland lesions than those without, indicating that allergies are related to different clinical phenotypes. The demographic features of our PAO/PA patients were consistent with those in other studies $[5,25,26]$. The shorter disease duration indicated that the severe disease activity needs urgent treatment, such as severe constitutional symptoms, back pain, higher inflammatory parameters, or renal function impairment due to ureter obstruction. Whether the low rate of allergy history of PAO/PA patients compared with those without suggested different triggering factors or etiology remained to be further elucidated.

The onset symptoms of PAO/PA patients were nonspecific, including pain (often back or abdominal pain, as chest or groin pain was relatively rare seen), edema of the lower limbs, and dyspnea [27]. In our cohort, the most common onset symptoms were back pain and abdominal pain, followed by lymph node swelling, submandibular gland enlargement, and lower limb edema in most patients with multiorgan involvement. However, back pain or abdominal pain is an atypical symptom, so abdominal CT or MRI must be conducted in patients with such symptoms for early detection of $\mathrm{PAO} / \mathrm{PA}$. Of $\mathrm{PAO} / \mathrm{PA}$ patients, the most prevalent type of vessel distribution was type 2, consistent with Ozawa et al.'s study [5, 26]. However, PAO/PA patients had a much lower rate of DS and paranasal sinusitis than non$\mathrm{PAO} / \mathrm{PA}$ patients, which is consistent with our previous study finding that patients without DS had a higher percentage of aorta or larger blood vessel involvement [7]. Nearly half IgG4-related PAO/PA patients in our cohort developed renal function impairment due to obstruction of the ureter. The percentage of creatinine elevation was higher in type 2 , especially in type $2 \mathrm{~b}$, indicating that urgent treatment (surgical intervention if necessary) may prevent those patients from experiencing deteriorating renal function.

Elevation of ESR and hsCRP was an indicator of vascular wall inflammation in large-vessel vasculitis, such as chronic periaortitis and TA [5, 26, 28, 29]. It has been reported that higher level of CRP is a hallmark for detecting perivascular involvement in IgG4-RD $[25,28]$. The mechanism of CRP elevation in IgG4-related PAO/ PA needs to be further elucidated. Our data demonstrated that PAO/PA patients had a higher white blood cell count, ESR, and hsCRP, but lower blood hemoglobulin, serum IgG4, and IgE levels than IgG4-RD patients without $\mathrm{PAO} / \mathrm{PA}$. Besides, in patients with only $\mathrm{PAO} /$ PA involvement, serum IgG4 levels were also lower than those in patients with PAO/PA and other organ involvement [7]. In our cohort, more than $70 \%$ of PAO/PA patients had elevated ESR and hsCRP, which is much higher than that reported by Mizushima et al., in which only a small proportion of patients had elevated hsCRP [26]. Patients with only PAO/PA involvement also had low levels of $\mathrm{CD} 19^{+} \mathrm{CD} 24^{-} \mathrm{CD} 38^{\text {hi }}$ plasmablast/plasma cells compared with patients with $\mathrm{PAO} / \mathrm{PA}$ and other organ involvement or non-PAO/PA patients. Circulatory inflammation was prominent, but low serum IgG4 in $\mathrm{PAO} / \mathrm{PA}$ patients compared with non-PAO/PA patients, indicating different disease pathogenesis that needs to be elucidated. Dacryoadenitis, higher serum IgG4, T-IgE, and higher circulating plasmablasts were risk factors for disease relapse [30-33], and the above parameter was lower in PAO/PA patients, which may indicate a lower relapse rate.

IgG4-RD with $\mathrm{PAO} / \mathrm{PA}$ might be a distinct spectrum of IgG4-RD, as it is characterized by prominent fibrosis, sparse lymphoplasmacytic infiltration, fewer extra-nodal germinal centers, and mildly elevated serum IgG1, IgG4, and IgE concentrations [5, 28, 34]. Recently, a pioneer approach to classifying IgG4-RD into proliferative 
subtype and fibrotic subtype was reported [35]. The characteristics of the fibrotic subtype usually involve extra-glandular sites and can involve a body region rather than a specific organ, including retroperitoneum fibrosis, sclerosing mesenteritis, and fibrosing mediastinitis. By comparison of IgG4-RD without PAO/PA in clinical manifestations, organ involvement, and laboratory parameters, IgG4-related PAO/PA patients showed distinct characteristics that could be classified into the fibrotic subtype.

Aortitis needs to be treated urgently because inflammatory aortic aneurysms may have a large diameter or high enlargement rate and are at a high risk of rupture. Patients with chronic periaortitis were often treated with medium to high dose GCs [14, 27]. Immunosuppressants are steroid-sparing treatments for PAO/PA patients. Previous studies indicated that $\mathrm{CYC}$ and MMF and rituximab were effective as induction therapy [10, 22, 36, 37]. For patients with ureter obstruction, quick relief from the obstruction by intra-ureteral stenting with a D-J stent could prevent further kidney damage. If obstruction is absent or mild and there is no renal function impairment, an immunosuppressive regimen is the first option. When moderate to severe ureteral obstruction and/or renal impairment are present, ureteral drainage must be the priority, followed by immunosuppressive therapy [38]. In view of the report of ruptured aneurysms after GC therapy in the literature [39], we used the strategy of moderate dose GC $(0.5 \mathrm{mg} / \mathrm{kg})$ combination and immunosuppressive therapy for nine patients with aneurysmal dilatation. There was no significant change in the scope and diameter of dilatation after treatment, and no patient experienced aneurysmal rupture. In our cohort, most of the PAO/PA patients were treated with GCs combined with immunosuppressant, and more than $90 \%$ of patients achieved CR. More female $\mathrm{PAO} / \mathrm{PA}$ patients could not achieve reduction of perivascular soft tissues for $>30 \%$, suggesting that female patients tended to be more resistant to treatment than male patients. In addition, $71.0 \%$ of our patients with ureteral obstruction were successfully extubated with a median time of 6 months. The reduction of ESR, hsCRP, serum IgG4, and T-IgE levels was also an indicator of treatment efficacy. Compared with IgG4-DS patients, a higher proportion of $\mathrm{PAO} / \mathrm{PA}$ patients managed to achieve normal serum IgG4 and IgE levels after treatment [7].

This study had some limitations. First, this is a singlecenter study. Second, the follow-up time was relatively short. Third, PET-CT is a more sensitive imaging test in evaluating vascular lesions; however, due to its high cost, most of the patients did not undertake PET-CT. Fourth, a more comprehensive investigation of pathogenesis needs to be conducted.

\section{Conclusions}

Our study indicates that IgG4-related PAO/PA is distinct from non-PAO/PA in demographic features, organ involvement distribution, inflammatory markers, and serum IgG4 and IgE level. The most common affected vessel was the abdominal aorta, and most patients responded well with treatment. As IgG4-related PAO/ PA is a spectrum of the fibrosis subtype of IgG4-RD, disease relapse is less likely to occur in patients with IgG4related $\mathrm{PAO} / \mathrm{PA}$ than in those without $\mathrm{PAO} / \mathrm{PA}$. Our study's findings could promote the understanding of IgG4-related $\mathrm{PAO} / \mathrm{PA}$ in clinical characteristics and treatment efficacy.

\section{Supplementary information}

Supplementary information accompanies this paper at https://doi.org/10 1186/s13075-020-02197-w.

Additional file 1: Supplementary Figure 1. Improvement of eGFR of IgG4 -related PAO/PA with renal impairment after treatment $(n=43)$. eGFR, estimated glomerular filtration rate.

Additional file 2: Supplementary Table 1. Comparison of characteristics at baseline among four types of vessel involvement groups. Q1, quartile 1; Q3, quartile 3; SD, standard deviation; WBC, white blood cell; HgB, hemoglobin; PLT, platelet; EOS, eosinophils; ESR, estimated sedimentation rate; hsCRP, hypersensitive C-reactive protein; Ig immunoglobulin; C3, complement 3; C4, complement 4; Cr, creatinine; lgG4-RD RI, immunoglobulin G4-related disease responder index.

Additional file 3: Supplementary Table 2. Comparison of characteristics at baseline between the more than 70\% responder group (Group A) and less than 30\% responder group (Group B). Q1, quartile 1; Q3, quartile 3; SD, standard deviation; WBC, white blood cell; HgB, hemoglobin; PLT, platelet; EOS, eosinophils; ESR, estimated sedimentation rate; hsCRP, hypersensitive C-reactive protein; Ig, immunoglobulin; C3, complement 3; C4, complement 4; Cr, creatinine; IgG4-RD Rl, immunoglobulin G4-related disease responder index; T-Ig, total immunoglobulin.

\section{Abbreviations}

Ig: Immunoglobulin; IgG4-RD: Immunoglobulin G4-related disease; PAO/ PA: Aortitis/periaortitis and periarteritis; CT: Computed tomography; MRI: Magnetic resonance imaging; PET-CT: Positron emission tomography/ computed tomography; IgG4-RD RI: IgG4-RD responder index; D-J stent: Double J stent; ESR: Erythrocyte sedimentation rate; hsCRP: Hypersensitive C-reactive protein; IgG: Immunoglobulin G; GCs: Glucocorticoids; IM: Immunosuppressant; CYC: Cyclophosphamide; MMF: Mycophenolate mofetil; eGFR: Estimated glomerular filtration rate; TA: Takayasu arthritis; IgG4-DS: IgG4-related dacryoadenitis/sialadenitis; C3: Complement 3; C4: Complement 4

\section{Acknowledgements \\ Not applicable.}

\section{Authors' contributions}

L.P. and P.Z. designed the study, performed the Odata analysis, and wrote the manuscript. P.Z. conducted flow cytometry measurements and data analysis. J.L., Z.L., and H.L. collected the data. L.Z., X.W., F.T., X.L., H.G., and Y.F. participated in the case and data collection. L.Z. helped with radiographic diagnoses. Y.Z., X.Z., and F. Z helped optimize the research and proofread the paper. W.Z. and Y.F. designed and directed the study and revised the manuscript. The authors read and approved the final manuscript.

\section{Funding}

This work was supported by The National Key Research and Development Program of China [2016YFC0901500], CAMS Innovation Fund for Medical 
Sciences (CIFMS) [2017-I2M-3-001], and National Natural Science Foundation of China [81771757, 81571587, and 81601433].

\section{Availability of data and materials}

The dataset analyzed in this paper is available from the corresponding author on reasonable request, and with appropriate additional ethical approvals, where necessary.

\section{Ethics approval and consent to participate}

The study was approved by the Ethics Committee of Peking Union Medical College Hospital. All patients consented to this study and signed a written informed consent

\section{Consent for publication}

Yes, we obtained consent for publication from all authors in the manuscript.

\section{Competing interests}

The authors declare that they have no competing interests.

\begin{abstract}
Author details
'Department of Rheumatology, Peking Union Medical College Hospital, Chinese Academy of Medical Science \& Peking Union Medical College, Key Laboratory of Rheumatology and Clinical Immunology, Ministry of Education \& National Clinical Research Center for Dermatologic and Immunologic Diseases (NCRC-DID), Beijing, China. ${ }^{2}$ Department of Radiology, Peking Union Medical College Hospital, Chinese Academy of Medical Science \& Peking Union Medical College, Beijing, China. ${ }^{3}$ Department of Rheumatology, The First People's Hospital of Yangquan, Yangquan, China. ${ }^{4}$ Department of Nephrology, Peking Union Medical College Hospital, Chinese Academy of Medical Science \& Peking Union Medical College, Beijing, China. ${ }^{5}$ Department of Rheumatology, The Second Hospital of Hebei Medical University, Shijiazhuang, China.
\end{abstract}

Received: 6 March 2020 Accepted: 23 April 2020

Published online: 04 May 2020

\section{References}

1. Shiokawa M, Kodama Y, Sekiguchi K, et al. Laminin 511 is a target antigen in autoimmune pancreatitis. Sci Transl Med. 2018;10(453):eaaq0997.

2. Hubers $L M$, Vos $H$, Schuurman $A R$, et al. Annexin A11 is targeted by lgG4 and IgG1 autoantibodies in IgG4-related disease. Gut. 2018;67(4):728-35.

3. Martin-Nares E, Angeles-Angeles A, Hernandez-Molina G. Major salivary gland enlargement in lgG4-related disease is associated with multiorgan involvement and higher basal disease activity. Mod Rheumatol. 2020;30:1727

4. Deshpande V, Zen Y, Chan JK, et al. Consensus statement on the pathology of IgG4-related disease. Mod Pathol. 2012;25(9):1181-92.

5. Ozawa M, Fujinaga $Y$, Asano J, et al. Clinical features of lgG4-related periaortitis/periarteritis based on the analysis of 179 patients with lgG4related disease: a case-control study. Arthritis Res Ther. 2017;19(1):223.

6. Wallace ZS, Zhang Y, Perugino CA, et al. Clinical phenotypes of IgG4-related disease: an analysis of two international cross-sectional cohorts. Ann Rheum Dis. 2019;78(3):406-12.

7. Wang M, Zhang P, Lin W, et al. Differences and similarities between IgG4related disease with and without dacryoadenitis and sialoadenitis: clinical manifestations and treatment efficacy. Arthritis Res Ther. 2019;21(1):44.

8. Kamisawa T, Zen Y, Pillai S, et al. IgG4-related disease. Lancet. 2015; 385(9976):1460-71.

9. Palmisano A, Urban ML, Corradi D, et al. Chronic periaortitis with thoracic aorta and epiaortic artery involvement: a systemic large vessel vasculitis? Rheumatology (Oxford). 2015;54(11):2004-9.

10. Perugino CA, Wallace ZS, Meyersohn N, et al. Large vessel involvement by IgG4-related disease. Medicine. 2016;95(28):e3344.

11. Stone JR. Aortitis, periaortitis, and retroperitoneal fibrosis, as manifestations of IgG4-related systemic disease. Curr Opin Rheumatol. 2011;23(1):88-94

12. Castelein T, Coudyzer W, Blockmans D. IgG4-related periaortitis vs idiopathic periaortitis: is there a role for atherosclerotic plaque in the pathogenesis of IgG4-related periaortitis? Rheumatology (Oxford). 2015:54(7):1250-6.

13. Kim IY, Eun $Y H$, Jeong $H$, et al. Clinical characteristics and outcomes of 61 patients with chronic periaortitis including lgG4-related and non-lgG4related cases. Int J Rheum Dis. 2017;20(11):1751-62.
14. Vaglio A, Catanoso MG, Spaggiari L, et al. Interleukin-6 as an inflammatory mediator and target of therapy in chronic periaortitis. Arthritis Rheum. 2013; 65(9):2469-75

15. Umehara $\mathrm{H}$, Okazaki $\mathrm{K}$, Masaki $\mathrm{Y}$, et al. Comprehensive diagnostic criteria for IgG4-related disease (IgG4-RD), 2011. Mod Rheumatol. 2012; 22(1):21-30.

16. Umehara $\mathrm{H}$, Okazaki $\mathrm{K}$, Nakamura $\mathrm{T}$, et al. Current approach to the diagnosis of IgG4-related disease - combination of comprehensive diagnostic and organ-specific criteria. Mod Rheumatol. 2017;27(3):381-91.

17. Schanzer A, Greenberg RK, Hevelone N, et al. Predictors of abdominal aortic aneurysm sac enlargement after endovascular repair. Circulation. 2011; 123(24):2848-55.

18. Caspary L. Inflammatory diseases of the aorta. VASA. 2016;45(1):17-29.

19. Scheel PJ Jr, Feeley N. Retroperitoneal fibrosis. Rheum Dis Clin N Am. 2013; 39(2):365-81.

20. Wallace ZS, Khosroshahi A, Carruthers MD, et al. An international multispecialty validation study of the lgG4-related disease responder index. Arthritis Care Res. 2018;70(11):1671-8

21. Campochiaro C, Ramirez GA, Bozzolo EP, et al. IgG4-related disease in Italy: clinical features and outcomes of a large cohort of patients. Scand J Rheumatol. 2016:45(2):135-45.

22. Yunyun F, Yu P, Panpan Z, et al. Efficacy and safety of low dose mycophenolate mofetil treatment for immunoglobulin G4-related disease: a randomized clinical trial. Rheumatology (Oxford). 2019;58(1): 52-60.

23. Brito-Zeron P, Ramos-Casals M, Bosch $X$, et al. The clinical spectrum of IgG4related disease. Autoimmun Rev. 2014;13(12):1203-10.

24. Tomelleri A, Campochiaro C, Sartorelli S, et al. Gender differences in clinical presentation and vascular pattern in patients with Takayasu arteritis. Scand J Rheumatol. 2019:48:482-90

25. Yamamoto $H$, Sugiyama E, Serikawa M, et al. Clinical features and predictive value of serum inflammatory markers of perivascular involvement in immunoglobulin G4-related disease. Heart Vessel. 2017; 32(10):1176-85.

26. Mizushima I, Inoue D, Yamamoto M, et al. Clinical course after corticosteroid therapy in IgG4-related aortitis/periaortitis and periarteritis: a retrospective multicenter study. Arthritis Res Ther. 2014;16(4):R156.

27. Mizushima I, Kasashima S, Fujinaga Y, Kawano M, Ishizaka N. IgG4-related periaortitis/periarteritis: an under-recognized condition that is potentially life-threatening. Mod Rheumatol. 2019:29(2):240-50.

28. Kasashima S, Kawashima A, Kasashima F, Endo M, Matsumoto Y, Kawakami K. Inflammatory features, including symptoms, increased serum interleukin6 , and C-reactive protein, in IgG4-related vascular diseases. Heart Vessel. 2018;33(12):1471-81.

29. Keser G, Aksu K, Direskeneli H. Discrepancies between vascular and systemic inflammation in large vessel vasculitis: an important problem revisited. Rheumatology (Oxford). 2018:57(5):784-90.

30. Wang $L$, Zhang $P$, Wang $M$, et al. Failure of remission induction by glucocorticoids alone or in combination with immunosuppressive agents in IgG4-related disease: a prospective study of 215 patients. Arthritis Res Ther. 2018;20(1):65.

31. Wallace ZS, Mattoo H, Carruthers M, et al. Plasmablasts as a biomarker for IgG4-related disease, independent of serum IgG4 concentrations. Ann Rheum Dis. 2015;74(1):190-5.

32. Lin W, Zhang $P$, Chen $H$, et al. Circulating plasmablasts/plasma cells: a potential biomarker for IgG4-related disease. Ann Rheum Dis. 2017; 19(1):25.

33. Culver EL, Sadler R, Bateman AC, et al. Increases in lgE, eosinophils, and mast cells can be used in diagnosis and to predict relapse of lgG4-related disease. Clin Gastroenterol Hepatol. 2017:15(9):1444-52 e6.

34. Palmisano A, Maritati F, Vaglio A. Chronic periaortitis: an update. Curr Rheumatol Rep. 2018;20(12):80.

35. Zhang W, Stone JH. Management of IgG4-related disease. Lancet Rheumatol. 2019:1:e55-65.

36. Binder $M, U$ hl $M$, Wiech $T$, et al. Cyclophosphamide is a highly effective and safe induction therapy in chronic periaortitis: a longterm follow-up of 35 patients with chronic periaortitis. Ann Rheum Dis. 2012;71(2):311-2.

37. Yunyun F, Yu C, Panpan Z, et al. Efficacy of cyclophosphamide treatment for immunoglobulin G4-related disease with addition of glucocorticoids. Sci Rep. 2017;7(1):6195. 
38. Rossi GM, Rocco R, Accorsi Buttini E, Marvisi C, Vaglio A. Idiopathic retroperitoneal fibrosis and its overlap with lgG4-related disease. Intern Emerg Med. 2017;12(3):287-99.

39. Tajima M, Hiroi Y, Takazawa Y, Muraoka H, Iwata H, Yamashita H, et al. Immunoglobulin G4-related multiple systemic aneurysms and splenic aneurysm rupture during steroid therapy. Hum Pathol. 2014;45:175-9.

\section{Publisher's Note}

Springer Nature remains neutral with regard to jurisdictional claims in published maps and institutional affiliations.

Ready to submit your research? Choose BMC and benefit from:

- fast, convenient online submission

- thorough peer review by experienced researchers in your field

- rapid publication on acceptance

- support for research data, including large and complex data types

- gold Open Access which fosters wider collaboration and increased citations

- maximum visibility for your research: over $100 \mathrm{M}$ website views per year

At BMC, research is always in progress.

Learn more biomedcentral.com/submissions 